\title{
Connected Edge Monophonic Domination Number of a Graph
}

\author{
P. Arul Paul Sudhahar \\ Department ofMathematics, \\ Rani Anna Govt. College (W) \\ Tirunalveli-627 008,
}

\author{
M. Mohammed Abdul \\ Khayyoom \\ Department of Higher \\ Secondary Education, \\ Govt. HSS, Pandikkad, \\ 676521 Kerala, INDIA.
}

\author{
A. Sadiquali \\ Department of Mathematical \\ Science, \\ MEA Engineering College, \\ Perinthalmanna-679 325, \\ Kerala, INDIA.
}

\begin{abstract}
In this paper the concept of connected edge monophonic domination number of a graph is introduced. A set of vertices $M$ of a graph $G$ is a connected edge monophonic domination set (CEMD set) if it is edge monophonic set, a domination set of $G$ and the induced sub graph $\langle M\rangle$ is connected. The connected edge monophonic domination number (CEMD number) of $G, \gamma_{m_{c e}}(G)$ is the cardinality of a minimum CEMD set. CEMD number of some connected graphs are realized. Connected graphs of order $n$ with CEMD number $n$ are characterised.It is shown that for every pair of integers $m$ and $n$ such that $3 \leq m \leq n$, there exist a connected graph $G$ of order $n$ with $\gamma_{m_{c e}}(G)=m$. Also, for any positive integers $p, q$ and $r$ there is a connected graph $G$ such that $m(G)=p, m_{\mathrm{e}}(G)=q$ and $\gamma_{m_{c e}}(G)=r$. Again, for any connected graph $G, \gamma_{m_{c e}}(G)$ lies between $\frac{n}{1+\Delta(G)}$ and $n$.
\end{abstract}

\section{AMS Subject Classification 05C12, 05C05}

\section{Keywords}

Edge monophonic number, monophonic domination number, edge monophonic domination number, connected edge monophonic domination numbers.

\section{INTRODUCTION}

By a graph $G=(V, E)$ we consider a finite undirected graph without loop or multiple edges. The order and size of a graph are denoted by $m$ and $n$ respectively. For basic graph theoretic notations and terminology we refer to [4] and [5].For vertices $u$ and $v$ in a connected graph $G$, the distance $d(u, v)$ is the length of a shortest $u-v$ path in $G$. A $u-v$ path of length $d(u, v)$ is called $u-v$ geodesic. A chord of a path $P: u_{1}, u_{2} \ldots u_{\mathrm{n}}$ is an edge $u_{\mathrm{i}} u_{\mathrm{j}}$ with $j \geq i+2$. A $u-$ $v$ path is monophonic path if it is chord less path. A monophonic set of $G$ is a set $M \subset V(G)$ such that every vertex of $G$ is contained in a monophonic path of some pair of vertices of $M$ (See [2],[9] and [10]).

The degree of a vertex $v$ in $G$ is the number of edges incident with $v$. The maximum degree of $G$ is the maximum degree among all the vertices of $G$ and is denoted by $\Delta(G)$. The neighborhood of a vertex $v$ is the set $N(v)$ consisting of all vertices which are adjacent with $v$. A vertex $v$ is an extreme vertex if the sub graph induced by its neighbourhood is complete. A vertex $v$ in a connected graph $G$ is a cutvertex of $G$, if $G-v$ is disconnected. A vertex $v$ in a connected graph $G$ is said to be semi-extreme vertex of $G$ if $\Delta(<N(v)>)=|N(v)|-1$. A graph $G$ is said to be semi- extreme graph if every vertex of $G$ is a semi extreme vertex. Every extreme vertex is a semi extreme vertex. Converse need not be true. An acyclic connected graph is called tree.

A dominating set in a graph $G$ is a subset of vertices of $G$ such that every vertex outside the subset has a neighbour in it. The size of a minimum dominating set in a graph $G$ is called the domination number of $G$ and is denoted $\gamma(G)$ (See [6]). A monophonic domination set of $G$ is a sub set of $V(G)$ which is both monophonic and dominating set of $G$.The minimum cardinality of a monophonic domonation set is denoted by $\gamma_{m}(G)$. An edge monophonic set of $G$ is a subset $M \subset V(G)$ such that every edge of $G$ is contained in a monophonic path joining some vertices of $M$. The minimum cardinality among all the edge monophonic sets of $G$ is called edge monophonic number and is denoted by $m_{\mathrm{e}}(G)$.( See [3] and [8]).

A set of vertices of $G$ is said to be edge monophonic domination set or EMD set if it is both edge monophonic set and a domination set of $G$. The minimum cardinality among all the EMD sets of $G$ is called edge monophonic domination number and is denoted by $\gamma_{m_{e}}(G)$. If $G$ is a connected graph of order $\quad n \geq 3$ and $G$ contains exactly one universal vertex, then $m_{\mathrm{e}}(G)=n-1$. (See[1])

The Petersen graph is a 3-regular graph of order 10. A caterpillar is a tree of order 3 or more, the removal of whose end-vertices produce a path called spine. The graph $C_{4} \times K_{2}$ is denoted by $Q_{3}$ and is called 3-cube graph. More generally, we define $Q_{1}$ to $K_{2}$ and for $n \geq 2$, define $Q_{\mathrm{n}}$ to be $Q_{\mathrm{n}-1} \times K_{2}$ and are called n-cubes or hyper cubes. The GreenwoodGleason graph is a 16 vertex graph with 40 edges which is a 5-regular graph and Hamiltonian. It is also vertex-transitive and edge-transitive with girth 4 . A vertex $v$ is a universal vertex of a graph $G$ if $\operatorname{deg}(v)=n-1$.

\section{CONNECTED EDGE MONOPHONIC DOMINATION NUMBER OF GRAPHS}

2.1 Definition: A set $M$ of vertices of a graph $G$ is connected edge monophonic domination set (abbreviated as CEMD set) if it is (i) an edge monophonic set of $G$ (ii) a domination set of $G$ and (iii) the induced sub graph of $M$, $\langle M\rangle$ is connected. The minimum cardinality among all the CEMD set of $G$ is called CEMD number and is denoted by $\gamma_{m_{c e}}(G)$.

2.2 Example: Consider the graph given in Figure 01 


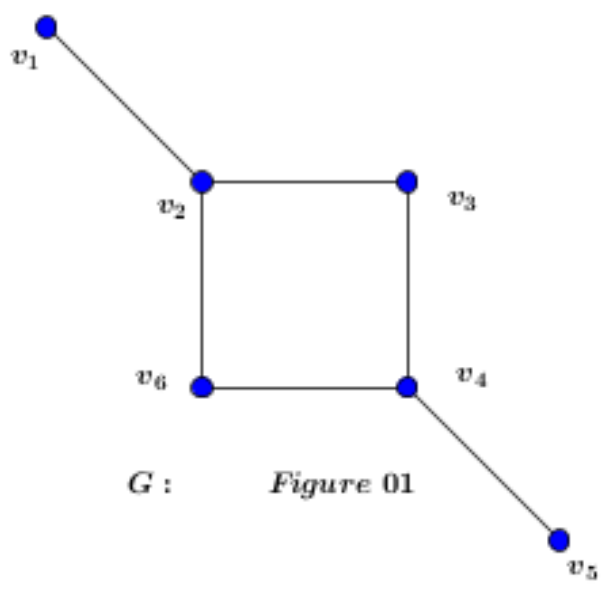

Here $M_{1}=\left\{v_{1}, v_{5}\right\}$ is an edge monophonic set, $M_{2}=\left\{v_{1}, v_{2}, v_{5}\right\}$ is an edge monophonic domination set and $M_{3}=\left\{v_{1}, v_{2}, v_{4}, v_{5}, v_{6}\right\}$ is a minimum CEMD set. Therefor $\gamma_{m_{c e}}(G)=5$.

2.3 Theorem: Let $G$ be a connected graph. Then $2 \leq$ $\gamma_{m_{e}}(G) \leq \gamma_{m_{c e}}(G) \leq n$.

Proof: Since any monophonic set contains at least two vertices, $2 \leq \gamma_{m_{e}}(G)$. Again, every CEMD set is an edge monophonic domination set, $\gamma_{m_{e}}(G) \leq \gamma_{m_{c e}}(G)$. Since the set of all vertices of $G$ is always a CEMD set, $\gamma_{m_{c e}}(G) \leq n$.

2.4 Remark: The bonds in Theorem 2.3 are sharp. In the example given in figure $01, n=6, \gamma_{m_{e}}(G)=3$, $\gamma_{m_{c e}}(G)=5$.

2.5 Theorem: For any connected graph of order n, $2 \leq$ $m_{c e}(G) \leq \gamma_{m_{c e}}(G) \leq n$.

Proof: Every connected edge monophonic set has at least two vertices. Also, every CEMD set is a connected edge monophonic set.

2.6 Theorem: Each extreme vertex belongs to every CEMD set.

Proof: Since each extreme vertex belongs to every monophonic set (see Theorem 2.3 [10]), these extreme vertices also belongs to every CEMD set.

2.6.1 Corollary: Each end vertices of a connected graph $G$ belongs to every CEMD set. This is due to end vertices are also extreme vertices.

2.7 Theorem: Each semi- extreme vertex belongs to every CEMD set.

Proof: Each semi extreme vertex belongs to every connected edge monophonic set of $\mathrm{G}$ (see Theorem 2.4[9]). Also every CEMD set is connected edge monophonic set, the result follows.

2.8 Theorem: For complete graph $K_{\mathrm{p}}, \gamma_{m_{c e}}(G)=p$.

Proof: In a complete graph $G$ every vertex is an extreme vertex and results follows from theorem 2.6

2.9 Theorem: For complete bipartite graph $K_{\mathrm{m}, \mathrm{n}}$

$$
\gamma_{m_{c e}}(G)=\left\{\begin{array}{c}
2, \quad \text { if } m=n=1 \\
n, \quad \text { if } n \geq 2, m=1 \\
\min \{m, n\}+1, \text { if } m, n \geq 2
\end{array}\right.
$$

Proof: Case (i) is trivial. Here the graph is $K_{2}$. Case (ii). Here the graph is a tree. Every vertex is either an extreme vertex or a cut vertex. For case (iii) take $X=\left\{x_{1}, x_{2} \ldots x_{\mathrm{m}}\right\}, Y=$ $\left\{y_{1}, y_{2}, \ldots y_{\mathrm{n}}\right\}$ be a partition of $G$. Assume $m \leq n$. Consider $D=X$. Then $D$ is a minimum edge monophonic set (By Theorem 2.11 of [1]. But its induced sub graph is not connected. Take $M=D \cup\left\{y_{i}\right\}$ for $1 \leq i \leq$ $n$.Then $C$ is a minimum CEMD set. There for $\gamma_{m_{c e}}(G)=$ $|M|=m+1=\min \{m, n\}+1$.

2.10 Theorem: For cycle graph $C_{\mathrm{n}}, \quad \gamma_{m_{c e}}\left(C_{\mathrm{n}}\right)=$ $n-2$, for $n \geq 5$.

Proof: Take any consecutive $n-2$ vertices in $C_{\mathrm{n}}$. These vertices dominates $C_{\mathrm{n}}$. Also that set is a connected edge monophonic set. Therefor $\gamma_{m_{c e}}(G) \leq n-2$. Now if the vertices are not consecutive it is not connected. Any $n-3$ or less consecutive vertices not dominate $C_{\mathrm{n}}$. Thus $\gamma_{m_{c e}}(G) \geq$ $n-2$.

2.11 Theorem: Each cut-vertex of a connected graph belongs to every CEMD set of $G$.

Proof: By theorem 2.7 of [9], each cut vertex of a connected graph $G$ belongs to every minimum connected edge monophonic set of G.Since every CEMD set is an edge monophonic set, the result follows.

2.12 Theorem: For any non-trivial tree $T$ of order n, $\gamma_{m_{c e}}(G)=n$.

Proof: Since every vertex of $T$ is either a cut vertex or an end vertex, the result follows from Theorem 2.11 and corollary 2.6.1.

2.13 Example: Let $G$ be the Cartesian product of two graphs $P_{\mathrm{n}}$ and $K_{2}$. That is $G=P_{\mathrm{n}} \times K_{2}$. Then $\gamma_{m_{c e}}(G)=n$ for $n \geq 4$.

Proof: Let $P_{\mathrm{n}}=\left\{v_{1}, v_{2}, \ldots, v_{\mathrm{n}}\right\}$ and $K_{2}=\{x, y\}$. Then the set $\mathrm{M}=\left\{\left(v_{1}, x\right),\left(v_{2}, x\right) \ldots\left(v_{\mathrm{n}}, x\right)\right\}$ is a minimum CEMD set. Therefor $\gamma_{m_{c e}}(G)=n$.
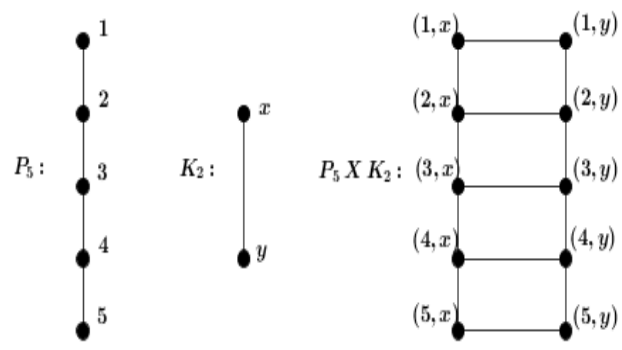

Figure 02 : Cartisian product to two graphs

\section{CEMD NUMBER OF SOME STANDERD GRAPHS}

\subsection{Peterson Graph:}

Consider the Peterson graph $G$ given in Figure 03. Here monophonic number, $m(G)=2$. Edge monophonic number $m_{\mathrm{e}}(G)=3$. Monophonic domination number $\gamma_{m}(G)=3$. Edge monophonic domination number $\gamma_{m_{e}}(G)=3$.CEMD 
number $\gamma_{m_{c e}}(G)=5$. The set $\left\{v_{1}, v_{2}, v_{3}, v_{4}, v_{5}\right\}$ is a minimum CEMD set.

\subsection{Caterpillar Graph:}

If $G$ is a caterpillar graph of $n$ vertices then $\gamma_{m_{c e}}(G)=n$. (See the Figure 04). Every vertices of caterpillar is either a cut vertex or an end vertex. The result follows from the theorem 2.12 .

\subsection{Hyper cube graphs:}

Consider the 3-cube graph $G=C_{4} \times K_{2}$ given figure 05 . $\gamma_{m_{c e}}(G)=4$. For a hyper cube graph $Q_{\mathrm{n}}, \gamma_{m_{c e}}\left(Q_{\mathrm{n}}\right)=2^{\mathrm{n}-1}$ for $n \geq 3$

\subsection{The Greenwood-Gleason graph:}

This is a five- regular connected graph with CEMD number 5.In Figure 06, the set $\left\{v_{6}, v_{7}, v_{8}, v_{9}, v_{10}\right\}$ is a minimum CEMD set.
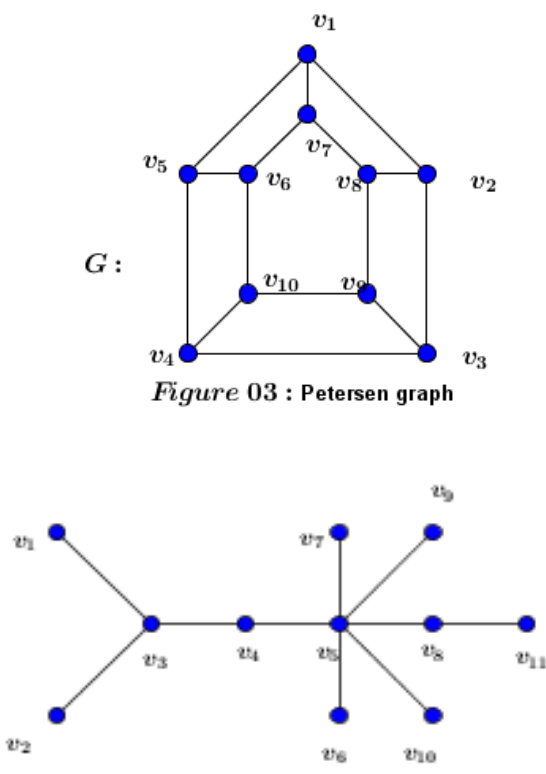

Figure 04: Caterpillar graph

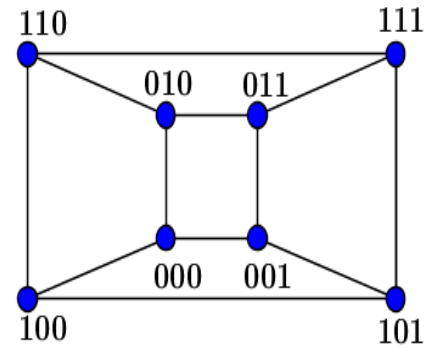

Figure 05 : 3-cube graph

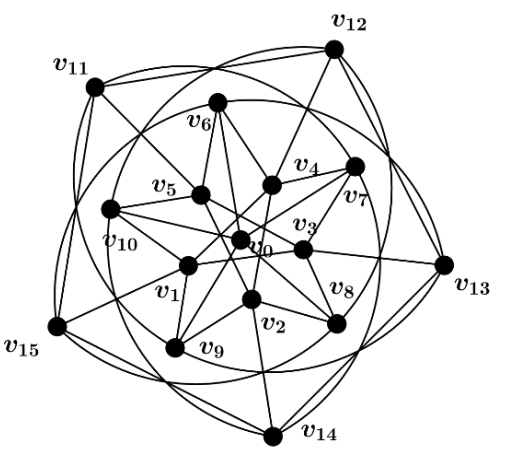

Figure 06 : The Greenwood-Gleanson Graph

\section{GENERALISATION RESULTS}

4.1 Theorem: Let $G$ be a connected graph. Then every vertex of $G$ is either a cut vertex or a semi-extreme vertex of $G$ if and only if $\gamma_{m_{c e}}(G)=n$

Proof: Since every cut vertex and semi extreme vertex belongs to every CEMD set, the necessary part is true. Conversely, let $\gamma_{m_{c e}}(G)=n$. Suppose there exist a vertex $p$ in $G$ which is neither a cut vertex nor a semi-extreme vertex. Since $p$ is not semi-extreme, the neighborhood of $p, N(p)$ does not induce a complete sub graph so that there exist two vertices $x$ and $y$ in $N(p)$ such that $d(x, y)=2$. That is $p$ lies on a $x-y$ monophonic path in $G$. Since $p$ is not a cut vertex of $G, G-p$ is connected. Thus $V(G)-\{p\}$ is a connected edge monophonic set of $G$. Since every $n-1$ vertices dominate $V(G)$, these vertices form a CEMD set which is a contradiction to the fact that $\gamma_{m_{c e}}(G)=n$. Hence $p$ is either a cut vertex or semi-extreme vertex.

4.2 Theorem: For every pair $m, n$ of integers with $3 \leq$ $m \leq n$, there exist a connected graph $G$ of order $n$ such that $\gamma_{m_{c e}}(G)=m$.

Proof: Let $P_{\mathrm{m}}: v_{1}, v_{2} \ldots, v_{\mathrm{m}}$ be a path of $m$ vertices. Take $n-m$ new vertices $x_{1}, x_{2} \ldots, x_{\mathrm{n}-\mathrm{m}}$ and join each $x_{\mathrm{i}}$ with $v_{1}$ and $v_{3}$, we get the connected graph $G$ (see the figure 07 ). Its order is $(n-m)+m=n$. Now let $D_{1}=\left\{v_{3}, v_{4} \ldots v_{\mathrm{m}}\right\}$. All these vertices are cut vertices of $G$ and belongs to minimum CEMD set. Thus $\gamma_{m_{c e}}(G) \geq m-2$. Neither $D_{1} \cup\left\{x_{i}\right\}$ nor $D_{1} \cup\left\{v_{2}\right\}$ are EMD set and $D_{1} \cup\left\{v_{1}\right\}$ is not connected. Thus $M=D_{1} \cup$ $\left\{v_{1}, v_{2}\right\}$ is a CEMD set and is minimum. Now $|M|=m-$ $2+2=m$ as desired 


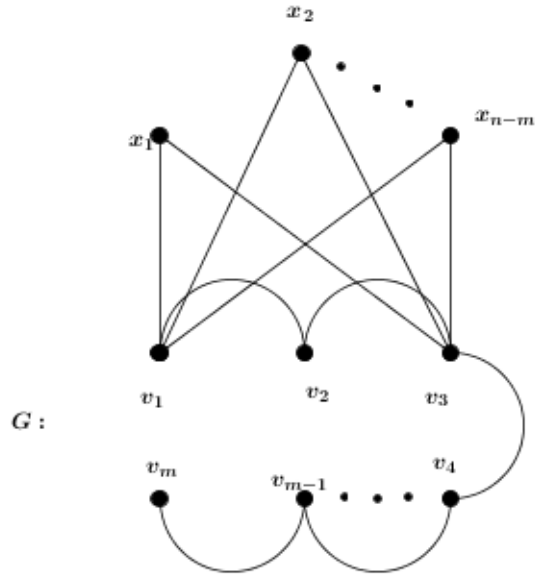

Figure 07

4.3 Theorem: Let $G$ be a connected graph of order $n$. Then $\frac{n}{1+\Delta(G)} \leq \gamma_{m_{c e}}(G) \leq n$, where $\Delta(G)$ is the maximum degree of $G$.

Proof: First, for the connected graph $G$, the set of all vertices is a CEMD set. Therefor $\gamma_{m_{c e}}(G) \leq n$. Suppose $\gamma_{m_{c e}}(G)=$ $k$. Take $M=\left\{v_{1}, v_{2} \ldots, v_{\mathrm{k}}\right\}$ be a minimum CEMD set. Next, any vertex $u_{\mathrm{i}}$ dominate $1+\operatorname{deg}\left(u_{\mathrm{i}}\right)$ vertices for $1 \leq i \leq$ $k$ and vertices of $M$ dominate all $n$ vertices of $G$. Therefor $n \leq \sum_{i=1}^{k} 1+\operatorname{deg} v_{\mathrm{i}}$. Now $\operatorname{deg} v_{\mathrm{i}} \leq \Delta(G)$. Therefor $1+\operatorname{deg} v_{\mathrm{i}} \leq 1+\Delta(G)$. That is $n \leq \sum_{i=1}^{k} 1+\operatorname{deg} v_{\mathrm{i}} \leq$ $\sum_{i=1}^{k} 1+\Delta(G) \leq k\left(1+\Delta(G)\right.$. Therefor $\mathrm{k} \quad \geq \frac{n}{1+\Delta(G)}$. Thus $\gamma_{m_{c e}}(G) \geq \frac{n}{1+\Delta(G)}$.

\section{CONCLUSION}

We can extent connected edge monophonic number to find upper connected EMD set, forcing connected EMD set and CEMD number of join of graphs, CEMD number of composition of graphs and CEMD hull number of graphs and so on. It has so many application in security of buildings and communication networks.

\section{REFERENCES}

[1] P. Arul Paul Sudhahar, M Mohammed Abdul Khayyoom and A Sadiquali. Edge Monophonic Domination Number of Graphs. J. Adv. in Mathematics. Vol 11. 10 pp 57815785 (Jan 2016)

[2] P Arul Paul Sudhahar, M Mohammed Abdul Khayyoom and A Sadiquali. Connected closed monophonic number of graphs. Indian J. Res. Found., (2016) 5, 17-21.

[3] P. Arul Paul Sudhahar, A. Sadiquali and M Mohammed Abdul Khayyoom. The Monophonic Geodetic Domination Number of Graphs. J. Comp. Math. Sci. Vol 7(1). Pp 27-38 (Jan 2016)

[4] F.Buckley, and F.Harary. Distance in Graphs, Addition Wesley, Redwood City, CA (1990):
4.4 Theorem: $\quad$ For any positive integer $p, q, r$ with $p \leq$ $q \leq r$ there exist a connected graph $G$ such that $m(G)=$ $p, m_{\mathrm{e}}(G)=q$ and $\gamma_{m_{c e}}(G)=r$.

Proof: Let $G$ be the graph given in Figure 08, having a path $x_{1}, x_{2}, x_{3} \ldots x_{\mathrm{r}-\mathrm{q}+2}$ and by adding $q-2$ vertices $y_{1}, y_{2} \ldots y_{\mathrm{q}-\mathrm{p}}$, $z_{1}, z_{2} \ldots z_{\mathrm{p}-2}$ with this path, and join each $y_{\mathrm{i}}$ with $x_{1}$ and $x_{3}$ and join each $z_{\mathrm{i}}$ with $x_{2}$. Then $M=\left\{z_{1}, z_{2} \ldots z_{\mathrm{p}-2}, x_{1}, x_{\mathrm{r}-\mathrm{q}-2}\right\}$ is a minimum monophonic set so that $m(G)=p$. Now $E=M \cup$ $\left\{y_{1}, \quad y_{2} \ldots y_{\mathrm{q}-\mathrm{p}}\right\}$ is a minimum edge monophonic set. Thus $m_{\mathrm{e}}(G)=|E|=q . \quad$ Take $\quad C=E \cup\left\{x_{2}, x_{3} \ldots x_{\mathrm{r}-\mathrm{q}+1}\right\}$. Cleary $C$ is a CEMD set and is the minimum. Hence $\gamma_{m_{c e}}(G)=|C|=q+(r-q+1)-1=r$.

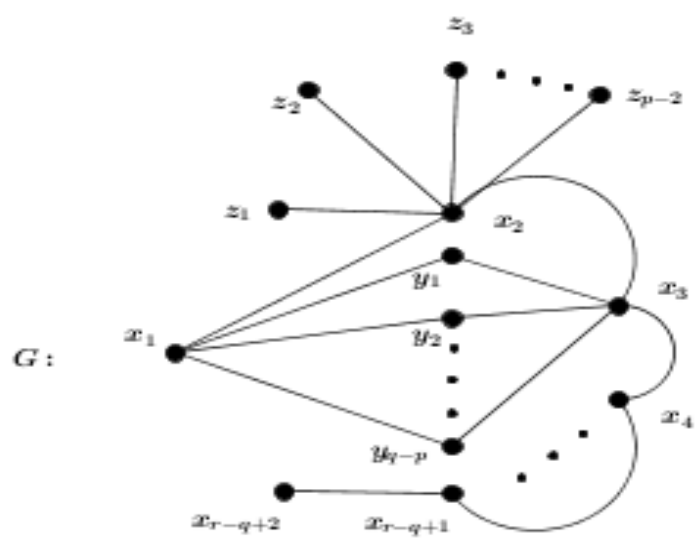

Figure 08

[5] Gary Chartrand and P.Zhang. Introduction to Graph Theory. Mac Graw Hill (2005)

[6] T W Haynes, S.T Hedetniemi and P.J Slater, Fundementals of Domination in Graphs,208, Marcel Dekker Inc, New York, 1998

[7] J. Jhon and P.Arul Paul Sudhahar. On The Edge Monophonic Number of a Graph. Filomat. Vol.26.6 pp 1081-1089(2012).

[8] J. Jhon and P.Arul Paul Sudhahar. The Monophonic Domination Number of a Graph. Proceedings of the International Conference on Mathematics and Business Management. (2012) pp 142-145.

[9] J.Jhon and P.Arul Paul Sudhahar. The Connected Edge Monophonic Number of a Graph. J. Comp. and Math. Sci. Vol 3(2), 131-136 (2012)

[10] A.P Santhakumaran, P. Titus and R. Ganesamoorthy.On The Monophonic Number of a Graph Applied Math and Informatics. Vol 32,pp 255-266 (2014). 